Peer-Reviewed Article

ISSN: 2162-3104 Print/ ISSN: 2166-3750 Online

Volume 7, Issue 3 (2017), pp. 825-840

(C) Journal of International Students

http://jistudents.org/

doi: 10.5281/zenodo.570036

\title{
Internet Use and Psychological Wellbeing: A Study of International Students in Singapore
}

\author{
Oindrila Dutta \\ Stefanie Yen Leng Chye \\ National Institute of Education, NTU, Singapore
}

\begin{abstract}
We investigated the relationship between psychological wellbeing (as indicated by participants' level of loneliness, perceived academic stress and depression) and generalized problematic internet use. Data was collected from a sample of 103 international students studying in Singapore. Statistical analyses revealed that depression was the most important predictor of problematic internet use, followed by academic stress. Loneliness was found to mediate the relationship between problematic internet use and depression or academic stress. Implications of the findings for international students in the University context have been discussed.
\end{abstract}

Keywords: academic stress, depression, loneliness, international students, psychological wellbeing

Several studies have highlighted the relationship between psychological well-being and excessive internet use (Ang, Chong, Chye, \& Huan, 2012; Caplan, 2002; Chong, Chye, Huan, \& Ang, 2014; Huan, Ang, Chong, \& Chye, 2014). However, these studies are not focused on students who travel overseas to pursue academics. Specifically, studies focusing on international students, studying in a university away from their country of origin have not been found. Travelling to a foreign country in pursuit of education requires students to adjust to environmental demands such as changes in their social network, acquiring non domain-specific life skills and meeting up to the academic expectations of the new environment. This restructuring can be especially traumatic for individuals who find it difficult to cope with these new exigencies, thus resulting in feelings of loneliness, depression and a sense of being unable to manage the stress (Stoliker \& Lafreniere, 2015; Thurber \& Walton, 2012). 


\section{LITERATURE REVIEW}

Similar to Davis (2001), it was found appropriate to use the phrase Problematic Internet Use (PIU) instead of Internet Addiction to refer to behaviors such as compulsively checking emails and cyber chats, responding to them throughout the day, procrastinating by involving oneself with different dimensions of the online world while simultaneously putting off other tasks and piling on pressures in other areas of life. The term Psychological wellbeing, in this study, referred to both the positive and negative emotions that the individual was experiencing as well as their subjective evaluation of the extent to which they felt satisfied with their life at a cognitive level (Diener \& Suh, 1997). The present study adhered to this definition and focused specifically on the negative aspects of psychological wellbeing, namely, loneliness, academic expectations stress and depression.

\section{Poor Psychological Wellbeing and Problematic Internet Use in International Students}

There is evidence which demonstrates the association between poor psychological wellbeing and problematic internet use (Caplan, 2002; Douglas et al., 2008; Morahan-Martin, 2003), more so in the case of younger users rather than older ones. This because younger users find it more di cult to manage multiple roles and demands on their time. This finding is especially important keeping in mind that the present research has as its focus group students from a university setting and potential behavioral concerns such as immoderate time spent online.

There also exists evidence pointing specifically to the presence of excessive and problematic internet use in the case of international students who struggle to meet their needs for social support within the physical constraints of their new environment (Dao, Lee, \& Chang, 2007). According to Caplan (2002), the internet offers its users a sense of anonymity which often functions as a kind of safety covering when interacting on platforms that comprise strangers or individuals with whom one isn't comfortable interacting face-to-face. This is especially enticing to individuals who perceive the demands of face-to-face communication to be stressful or perceive themselves to be lacking in the required competencies since they perceive it as a kind of functional alternative for social communication and interaction.

Loneliness, that is, a discrepancy between ones desired and achieved level of social contact (Peplau \& Perlman, 1979) is closely related to problematic internet use, which can translate into negative consequences in various domains of life (Caplan, 2002; Douglas et al., 2008). Events which change an individual's desired and/or achieved level of social contact may serve as predisposing factors to loneliness (Dao et al., 2007). This puts 
overseas students who are distanced from the comforts of their social support system at an increased risk for loneliness. And, as research has found, lonely individuals are more likely to resort to internet use as a means of seeking social support (Morahan-Martin, 2003).

Past research has documented the association between depression and problematic internet use (Young, 1998; Young \& Rogers, 1998). Evidence also indicates that depression levels are significantly high amongst international students pursuing academic activities away from their home country (Han, Han, Luo, Jacobs, \& Jean-Baptiste, 2013; Mesidor \& Sly, 2015; Rice, Choi, Zhang, Morero, \& Anderson, 2012; Sa, Seo, Nelson, \& Lohrmann, 2013). Taken together, these studies suggest that international students are at risk of depression and problematic internet use.

Maladaptive thoughts and negative emotions from academic-related stress has been found in research to be an important contributor to problematic internet use (Chong et al., 2014; Jun, 2015). However, no data was found regarding the relationship between problematic internet use and academic stress amongst overseas students. Yet, it could be possible that such a relationship exists since overseas students in Singapore are a population that is predominantly Asian in nature. The unique cultural experience of such a sample would be the heavy emphasis on academic achievement since a young age and expectations from both the home and school environment to excel academically, which is more deeply experienced than individuals raised in cultures of the West or individuals of Asian descent but currently living in the Western world (Chong et al., 2014). Aconcerning factor is the finding that individuals who have wider access to the internet are more likely to become addicted than those who did not have ease of access (Young \& Rogers, 1998). Within the local context of Singapore and especially within the geographical limits of university education, internet connection and access is of a superior quality and conveniently accessible (Teo, Lim, \& Lai, 1997).

The present study has the following research questions:

1. What are the relationships between loneliness, depression, academic expectations stress and problematic internet use?

2. Does loneliness, depression, and academic expectations stress predict problematic internet use? To what extent?

\section{RESEARCH METHOD}

Data were collected from 103 international students (56 males and 47 females) studying in Singapore's Nanyang Technological University through the convenience method of sampling. Self-reported ethnic identification of the participants was $64.1 \%$ Indian, $15.5 \%$ Chinese, $6.8 \%$ Asian (other), $4.9 \%$ Caucasian and 1\% African. $62.1 \%$ of participants were 
in the age range of $21-25$ years, $35.9 \%$ in the age range of $26-30$ years and $1.9 \%$ in the age range of $31-35$ years. $50.5 \%$ of participants were master's students and the remaining were doctoral candidates. $95.1 \%$ of participants were unmarried. The number of years that the participants had been pursuing their graduate studies in Singapore ranged from 1-6 years (which is the minimum to maximum period of candidature in the University).

Ethical consent for the research was secured from the Institutional Review Board of the University. Students were approached by the researchers both via an online link to the surveys, as well as in friendly environments. Participant were provided a brief description of the study procedures and informed that participation was voluntary. All questionnaires were administered in English. No translation was required since English is the medium of instruction in Singapore's Nanyang Technological University.

\section{Measures}

Personal Information Form. Participants were requested to provide demographic data about themselves such as sex, age, ethnicity, marital status and educational level in order for the researcher to better understand the data obtained from the surveys and make more meaningful analyses.

Depressive Attributions Questionnaire. The Depressive Attributions Questionnaire (DAQ) was a 16 item self-report measure which assessed the extent to which an individual had a pessimistic or depressogenic attributional style, that is, a tendency to attribute negative outcomes to internal, stable and global factors (Kleim \& Gonzalo, 2011). Research (Luten, Ralph, \& Mineka, 1997) has found that such pessimistic cognitive patterns are a significant predictor of depression. Participants in the present study were required to assess each of the DAQ items on a 5 point Likert scale ranging from strongly disagree (0) to strongly agree (5) such that a higher score indicates stronger endorsement of depression. Thus, higher the score, greater would be the level of depressive attributions in participants. Sample items from the survey include: When bad things happen, I think it is my fault; when something good happens, I think it will not last long. In the present study, the range of scores for all items was between 1 and 5 except item 10 for which the scores ranged between 1 and 4. Mean participant scores on the items ranged from 1.92 to 3.08. The standard deviations of participant scores on the items ranged from 0.87 to 1.20. For this study, the Cronbach alpha reliability estimate obtained was .857 .

Academic Expectations Stress Inventory. The Academic Expectations Stress Inventory (AESI) was a 9 item self-report questionnaire that comprised 5 items which measured academic stress perceived by Asian 
students from their parents or teachers (for example, I blame myself when I cannot live up to my parents' expectations of me) and 4 items which measured academic stress perceived by Asian students from themselves (for example, I feel stressed when I do not live up to my own standards) (Ang \& Huan, 2006). Responses could lie across a 4 point Likert scale which ranges from never true (1), almost never true (2), almost always true (3) and always true (4). Thus, higher the score, greater would be the perceived stress from academic expectations. In the present study, the range of scores for all items was between 1 and 4 . In this study, mean participant scores ranged from 2.27 to 3.25 . The standard deviations of participant scores on the items ranged from 0.75 to 1.03 . The Cronbach alpha reliability estimate obtained for this scale was .826 .

UCLA Loneliness Scale. The University of California, Los Angeles Loneliness Scale (UCLA Loneliness Scale) was a 20 item measure which assessed an individual's subjective feelings of loneliness and social isolation (Russell, 1996). Item responses on the UCLA Loneliness Scale could range from never (1) to often (4) such that higher scores would indicate greater levels of loneliness in participants. Thus, higher the score, greater would the level of loneliness indicated by participants. Sample items from the survey include: I am unhappy doing so many things alone; I find myself waiting for people to call or write. In the present study, mean participant scores on the items ranged from 1.88 to 2.59 . The standard deviations of participant scores on the items ranged from 0.82 to 1.07 . The Cronbach alpha reliability estimate obtained for this scale was .944.

Generalized Problematic Internet Use Scale. According to Caplan (2002), the Generalized Problematic Internet Use Scale (GPIUS) was a 29 item self-report measure which could be useful in identifying the extent to which a person experienced the kind of thoughts, behaviors and consequences that were generally associated with internet usage. Responses could typically lie across a 5 point Likert scale which ranged from strongly agree (1) to strongly disagree (5), where higher scores would indicate problematic thinking, behavior and outcomes associated with excessive internet use. Thus, higher the score, greater would be the participants' indication of problematic internet use. Sample items from the survey include: I use the Internet to make myself feel better when I'm down; 7. I am more confident socializing online than offline. In the present study, the mean participant scores on the items ranged from 1.71 to 3.51. The standard deviations of participant scores on the items ranged from 0.75 to 1.31 . The Cronbach alpha reliability estimate obtained for this scale was .906 .

\section{Data Analytic Procedures}

The data was collected in a spreadsheet and re-organized for analysis using the IBM SPSS Statistics 23. Cronbach alpha was also 
calculated for the psychometric instruments employed in the study as a means to determine the internal reliability of the scales used. The scales used in this study all yielded alpha values greater than 0.7 suggesting good internal reliabilities. Similar to the design employed by Morrison and Gore (2010), Pearson Product Moment Correlation Coeffcients were computed to examine the relationships between scores on the (i) UCLA Loneliness Scale; (ii) Academic Expectations Stress Inventory (AESI); (iii) the Depressive Attributions Questionnaire (DAQ); and (iv) the Generalised Problematic Internet Use Scale (GPIUS).

To examine the predictive relationships of loneliness, academic expectations stress and depressive attributions with GPIU, a hierarchical multiple regression was computed. A sample size of 103 was deemed adequate in view of the 3 independent variables to be included in the analysis (Keith, 2014; Tabachnick \& Fidell, 2007). Subsequently, a two-step hierarchical regression was conducted with GPIU as the dependent variable. The demographic variables of gender, age, ethnicity, marital status and educational status were entered at stage 1 of the regression to control for the impact of these demographic variables. Loneliness, Academic Expectations Stress, and Depressive Attributions were entered at stage 2 of the regression.

\section{RESULTS}

Results obtained from the correlational analyses are reported in Table 1.

Table 1: Descriptive statistics and Pearson's correlations of Depression, Loneliness and Academic Expectations stress (AES) and GPIU.

\begin{tabular}{llllllll}
\hline & $\mathrm{N}$ & Mean & Stdev & Depression & Loneliness & AES & GPIU \\
\hline Depression & 103 & 2.48 & .58 & - & - & - & $.364^{* *}$ \\
Loneliness & 103 & 2.15 & .67 & - & - & - & $.287^{* *}$ \\
AES & 103 & 2.64 & .62 & - & - & - & $.404^{* *}$ \\
GPIU & 103 & 2.58 & .57 & $.364^{* *}$ & $.287^{* *}$ & $.404^{* *}$ & - \\
\hline
\end{tabular}

Table 1, shows a significant correlation between loneliness and generalized problematic internet use (GPIU). It can be observed that $r=.287$, $\mathrm{p}<0.01$. For international students studying in Singapore then, there is evidence that problematic use of the internet is strongly related with their levels of loneliness. In particular, it seemed that students who were lonelier and socially isolated tended to report higher levels of problematic internet use. From Table 1, it can be observed that there is a significant correlation between depression and GPIU, where $r=.364, \mathrm{p}<0.01$. This suggests that for international students studying in Singapore, problematic use of the internet is strongly related with their levels of depression. In particular, it seemed that students who were more depressed tended to report higher levels of problematic internet use. From Table 1, it can be observed that there is a significant correlation between academic expectation stress (AES) and 
(GPIU) where $\mathrm{r}=.404, \mathrm{p}<0.01$. For international students studying in Singapore then, there is evidence that problematic use of the internet is strongly related with their levels of academic expectation stress. In particular, it seemed that students who were more stressed about meeting up to expectations with respect to their academics tended to report higher levels of problematic internet use.

Table 2: Summary of Hierarchical Regression Analysis for Variables Predicting GPIU ( $\mathbf{N}=\mathbf{1 0 3})$

\begin{tabular}{lllll}
\hline Model & & \multicolumn{2}{l}{ Unstandardized coefficients } & $\begin{array}{l}\text { Standardized } \\
\text { coefficients } \\
\text { Beta }\end{array}$ \\
\cline { 2 - 5 } 1 & B & Std. Error & 11.713 & \\
& (Constant) & 65.286 & 3.348 & 0.02 \\
Sex & 0.619 & 3.027 & -0.145 \\
Age & -4.321 & 0.882 & 0.125 \\
Ethnicity & 1.061 & 7.258 & 0.025 \\
Marital status & 1.844 & 3.269 & 0.157 \\
Edu status & 4.96 & 13.352 & \\
(Constant) & 24.367 & 3.039 & -0.046 \\
Sex & -1.457 & 2.836 & -0.038 \\
Age & -1.137 & 0.807 & 0.103 \\
Ethnicity & 0.872 & 6.564 & 0.025 \\
Marital status & 1.861 & 2.961 & 0.179 \\
Edu status & 5.651 & 0.295 & $.280^{* *}$ \\
AES total & 0.801 & 0.192 & $.291 *$ \\
Depression & 0.496 & & \\
total & & 0.134 & 0.003 \\
Loneliness & 0.003 & & \\
total & & &
\end{tabular}

The hierarchical multiple regression revealed that at Stage 1, the demographic variables contributed $6.3 \%$ to the regression model but this contribution was not significant, $F(5,97)=1.298, p>.05$. Introducing the psychological well-being variables as a block, explained an additional $20.4 \%$ of the variation in GPIU and this change was significant. F $(3,94)=$ $8.700, \mathrm{p}<.01$. When all independent variables were included in Stage 2 of the regression model, the most important predictor of GPIU was Depression $(\beta=.291, \mathrm{p}<.05)$, followed by Academic Expectations Stress (AES) $(\beta$ $=.280, \mathrm{p}<.01)$. Loneliness was not a significant predictor of GPIU $(\beta=.003$, $\mathrm{p}>.05)$.

\section{DISCUSSION AND CONCLUSIONS}

The present study investigated the relationship between Generalized Problematic Internet Use (GPIU) on one hand and aspects of psychological 
wellbeing such as loneliness, depression and academic expectation stress on the other, in a sample of international students studying in Singapore. The study obtained positive and significant associations between loneliness and GPIU. The international students studying in Singapore who were lonelier and socially isolated tended to report higher levels of problematic internet use. These findings are in line with those of Morahan-Martin \& Schumacher (2003), who opined that lonely individuals resorted to internet use as a means of seeking emotional support, making online friends and coping with their negative moods which interfered with their everyday functioning. Research is of the opinion that once students traversed the physical boundaries of their home country to pursue education elsewhere, they ceased to belong to the world they had left behind, but neither did they belong to the new world into which they had recently arrived (Grinberg \& Grinerg, 1990). They found themselves negotiating a foreign language, a different kind of scholastic environment as well as day-to-day concerns such as finances and accommodation. At a time when social support would be most appreciated, international students tended to find themselves in a state of relational deficit. Many of them revisited the stage of identity crisis having to recreate their personal autonomy within the new social boundaries. In this way, both routine and profound loneliness were an integral part of the international student experience.

Data obtained in this study revealed a positive and significant relationship between Depression and GPIU. Students who were more depressed tended to report higher levels of problematic internet use. The evidence obtained is in line with past research which has identified an association between excessive internet use resulting in significant social, psychological, and occupational impairment (Shaw \& Black, 2008; VandeCreek, 2000). More recently, it was found that there was a high correlation between scores on the Beck Depression Inventory and signs of internet dependency (Morrison \& Gore, 2010). Given that participants in the present study were international students among whom depression levels would be significantly high (Rice et al., 2012; Sa et al., 2013), the positive correlation between scores on the Depressive Attributions Questionnaire and scores on the Generalized Problematic Internet Use Scale confirmed the expected association between these two variables.

Results in the present study were indicative of a positive and significant correlation between scores on the Academic Expectations Stress (AES) and scores on the GPIU. Students who were more stressed about meeting academic expectations tended to report higher levels of problematic internet use. In their study, Chong et al. (2014) explained similar findings in terms of parenting practices in Asian cultures. They suggested that in Asian cultures, there was a lot of focus on academic achievement in both the family and school settings. High expectations were placed on students who 
would be likely to measure their filial piety and self-worth based on the extent to which they saw themselves as meeting up to these expectations. This urge to do well and prevent loss of face would be a major source of psychological stress for students, which would be similar to the demands experienced by students in other parts of the world. But the distinguishing factor in Asian cultures, according to these researchers was that the stress arose majorly from authority figures and significant others within the family rather than from the self. The authors further elaborated that adolescents with unrealistic scholastic achievement expectations could form dysfunctional cognitive patterns which would lead them to fall into a web of anxiety that they might disappoint their significant others if they are incapable of meeting up to these perceived demands.

Past literature suggested that adjusting to college life was difficult for all students because of the change in their scholastic and social surroundings (Misra, 2004). To add to this, overseas students had the pressure to gain fluency in the language and cultural values of the host country. With the snowballing of environmental stressors, students and especially those from foreign cultures might perceive it as exceeding their existing coping resources. This would in turn increase their chances of developing both physical and psychological reactive symptoms (Lazarus, 1984) thus increasing their predispositions to excessive use of the internet.

Another aim of the present study was to examine whether and the extent to which loneliness, depression and AES predicted GPIU. After controlling for the potential effects of demographic variables, it was established that demographic variables contributed $6.3 \%$ to GPIU but this contribution was not significant. On the other hand, introducing psychological well-being variables explained an additional $20.4 \%$ of the variation in GPIU and this change was significant. These findings suggest that psychological wellbeing are important contributors to GPIU.

In addition, the results revealed that the most important predictor of GPIU was Depression, followed by Academic Expectations Stress. Loneliness was found not to be a significant predictor of GPIU. Risk factors such as the existence of depressive symptoms can predispose an individual to become a problematic user of the internet (Davis, 2001). It is possible that such individuals operate the internet as a means to reduce or escape from their psychological distress (Caplan, 2002; Davis, 2001). This in turn can result in online overuse of a problematic nature (Davis, 2001). Other research has explained this phenomenon as a consequence of an individual's depressive mood interfering with his ability to self-regulate his thinking and behavior. Problematic internet use is developed as a consequence of this inability (LaRose \& Eastin, 2002). The present study contributes to the academic literature in that it establishes such a phenomenon of depression and its contributions to problematic internet use, specifically in the case of 
international students amongst whom depressive symptoms have been a cause of concern for mental health professionals (Han et al., 2013; Mesidor \& Sly, 2015; Rice et al., 2012; Sa et al., 2013).

There is evidence to suggest that stressful situations in an individual's life, especially during the years in college, can significantly contribute to the development of GPIU (Wang \& Wang, 2009). Wang \& Wang (2009) explained that such individuals, rather than confronting the stressors, might engage in avoidant ways of coping with their life stressors such as fantasizing, withdrawing and rationalizing, for which the internet provides plethora of opportunities. The findings of the present study are consistent with this line of thought and the academic dialogue focusing on the multitude of stressors that international students confront which includes navigating the new socio-cultural environment of the host country (Dao et al., 2007), and meeting up to the cultural expectations of scholastic achievement, which is especially unique to Asian samples of population, such as the sample in the present study (Chong et al., 2014).

Other studies have documented that there is a direct and significant relationship between loneliness and GPIU (Morahan-Martin, 2003), perhaps even stronger than the link between depression and GPIU (Caplan, 2002). However, similar results were not obtained in the present study. Specifically, it was found that the link between depression and GPIU and academic stress and GPIU were both positive and significant in nature, but the relationship between loneliness and GPIU was not significant. Since such results have not been obtained in past studies, it might be useful to investigate this relationship further. Perhaps some unique features of the present research such as the focus on international students within the setting of Nanyang Technological University, Singapore, might have differential effects on this link between loneliness and GPIU. For example, it could be hypothesized that vibrant student life within the campus of this University may protect students from falling victim to loneliness despite the perils of being an international student (Council, 2016; Nanyang Technological University, 2016; Union, 2016). There is evidence to show that when University management recognize international students' needs and offer relevant supportive on-campus resources and services, students feel embraced into the academic and social world of the University, which in turn enhances their adjustment to the host culture of the University (Wu, Garza, \& Guzman, 2015). No investigation was carried out to test this hypothesis but it could be a focus of future research, to fill this gap in evidence. It would also be interesting to investigate what other kinds of populations show evidence contrary to the persistent strong relationship that previous literature has suggested between loneliness and internet use.

Overall, the findings obtained in the present study were in line with the framework of Compensatory Internet Use (Kardefelt-Winther, 2014). 
According to Kardefelt-Winther (2014), certain individuals might spend excessive amounts of time online such as in playing online games as a way of coping with psychosocial problems. In their study, it was found that individuals who experienced high stress and low self-esteem levels tended to use the internet excessively as a strategy to escape from the struggles of everyday life. Thus the findings highlighted the link between poor psychological wellbeing and problematic internet use.

\section{IMPLICATIONS}

There is ample research evidence to support that maladaptive coping mechanisms are prevalent amongst international students (Constantine, Kindaichi, Okazaki, Gainor, \& Baden, 2005; Smith \& Khawaja, 2011; Wei, $\mathrm{Ku}$, Russell, Mallinckrodt, \& Liao, 2008). The findings obtained from these studies have important implications for counselors and especially those working within University settings that have a large international population. Based on a comprehensive review of literature in the area of coping of international students, research has highlighted that indirect coping mechanisms which do not rectify the immediate problem (for example, accepting the situation and positively reinterpreting it) was linked with lower levels of perceived stress and therefore lower levels of depression (Smith \& Khawaja, 2011). This highlights the usefulness of positive reframing when working with psychologically distressed overseas students struggling with internet use of a problematic nature. Some research (Smith \& Khawaja, 2011) has suggested that the Rogerian condition of unconditional positive regard (that is, compassion for oneself) amongst Chinese international students helped to significantly reduce the stress of adjusting to a new culture. Kennedy (1994, as cited in Smith \& Khawaja, 2011) put forth the idea that humor can be a positive coping strategy employed by overseas students to improve mood. Thus, it appears that one of the methods that might be useful in reducing problematic internet use as a function of loneliness, depression and academic expectation stress amongst overseas students is to enhance more positive coping strategies. Although direct research evidence is lacking, future research might focus on testing the effectiveness of implementing such functional coping strategies amongst students which, as previous research suggests, has the potential to reduce problematic coping styles.

Although transition to university is a novel and exciting experience for students and especially for those who step into adulthood by moving away from the home environment of their childhood (for example, international students who relocate to a new country in pursuit of their scholastic goals), it can also be a source of stress for many individuals, culminating in psychological concerns such as depression, loneliness and 
maladaptive ways of coping (Thurber \& Walton, 2012). However, given that acquiring post-secondary education is considered to be a developmental milestone in today's day and age, healthy adjustment to separation is essential for maximizing the educational and social benefits of the experience (Thurber \& Walton, 2012). Thurber and Walton (2012) are of the opinion that university policies should play a key role in undertaking preventive measures, that is, e orts prior to the arrival of students which will enhance their confidence in perceived ability to cope and generate more positive attitudes towards this experience of moving away from one's comfort zone. Some of their recommendations include:

- Establishing a sense of agency in making important decisions related to their University life such as choice of coursework, residence or schedule;

- Provide orienting information about the school, its environment, and its culture;

- Shaping attitudes about the school through Web-based and inperson discussion of the positive aspects of the experience;

- Initiating social contacts prior to the students' arrival so that the incoming students feel connected to and supported in the new environment.

\section{LIMITATIONS AND CONCLUSION}

When generalizing the findings of this study, it is important to keep in mind the following limitations which may pose questions upon its external validity to internet usage and psychological wellbeing of international students the world over. The sample consisted exclusively of international students from Singapore's Nanyang Technological University who are currently enrolled in either a master's or a doctorate program. Hence the application of these findings may be restricted by boundaries of physical space and education culture. Additionally, the sample consisted primarily of racially Indian students, followed by Chinese and other Asian races with a scarce Caucasian or non-Asian representation. Potential cultural differences may have biased the findings. Another limitation was that the investigation did not take into account whether the participants from overseas had previous experience of being an international student, or whether they had left their home country for the first time to pursue academics. It is hoped that subsequent research in this area will consider controlling this factor. The study was conducted using a survey methodology which may have resulted in participants being more concerned with social impression management rather than providing responses to generate accurate results and interpretations. Although the researcher did stress on anonymity of participants, it is unclear whether the participants themselves were assured. 
However, given the nature of the variables, it appears di cult to obtain data through a technique different from self-report inventories. Perhaps future research in this area might consider focusing on assessments by teachers, peers and significant others of the participants to obtain a more comprehensive view of the situation. Further, the methodology involved use of quantitative measures such as questionnaires which may be written and developed for limited cultures and cohorts which restricts their suitability when applied to other populations. Future research in this area might consider taking into account this factor of ensuring that the measures were appropriate to the demographics of the sample. The researcher did not have information about certain other potentially mediating variables, specifically participants' impulse control abilities which could have confounded the relationship between the suggested areas of psychosocial functioning and problematic internet use. The study was conducted on an overseas student population currently residing and pursuing graduate studies in Singapore and the results were not compared with those of a clinical population. It is recommended that prospective studies in this domain consider taking into account this aspect as well. Further, a cross sectional methodology comparing international graduate students with international undergraduate or high school students as well as with local graduate students would also be useful in narrowing down which quality of their experience is salient in the relationship with excessive internet use. Lastly, it might be of interest to follow the same participant students throughout their period of their study in the University. Developments observed in a longitudinal study could be interestingly different from those obtained at the given moment.

\section{REFERENCES}

Ang, R. P., Chong, W. H., Chye, S., \& Huan, V. S. (2012). Loneliness and generalized problematic Internet use: Parents' perceived knowledge of adolescents' online activities as a moderator. Computers in Human Behavior, 28(4), 1342-1347.

Ang, R. P., \& Huan, V. S. (2006). Academic expectations stress inventory development, factor analysis, reliability, and validity. Educational and Psychological Measurement, 66(3), 522-539.

Caplan, S. E. (2002). Problematic Internet use and psychosocial well-being: development of a theory-based cognitive-behavioral measurement instrument. Computers in Human Behavior, 18(5), 553-575.

Chong, W. H., Chye, S., Huan, V. S., \& Ang, R. P. (2014). Generalized problematic Internet use and regulation of social emotional competence: the mediating role of maladaptive cognitions arising from academic expectation stress on adolescents. Computers in Human Behavior, 38, 151-158.

Constantine, M. G., Kindaichi, M., Okazaki, S., Gainor, K. A., \& Baden, A. L. (2005). A qualitative investigation of the cultural adjustment experiences 
of Asian international college women. Cultural Diversity and Ethnic Minority Psychology, 11(2), 162.

Council, G. S. (2016). NTUGSC: Recent News and Events.

Dao, T. K., Lee, D., \& Chang, H. L. (2007). Acculturation level, perceived English fluency, perceived social support level, and depression among Taiwanese international students. College Student Journal, 41(2), 287.

Davis, R. A. (2001). A cognitive-behavioral model of pathological Internet use. Computers in Human Behavior, 17(2), 187-195.

Diener, E., \& Suh, E. (1997). Measuring quality of life: Economic, social, and subjective indicators. Social Indicators Research, 40(1-2), 189-216.

Douglas, A. C., Mills, J. E., Niang, M., Stepchenkova, S., Byun, S., Ruffini, C., ... others. (2008). Internet addiction: Meta-synthesis of qualitative research for the decade 1996-2006. Computers in Human Behavior, 24(6), 30273044.

Grinberg, L., \& Grinerg, R. (1990). Psychoanalytic perspectives on migration and exile. Choice Reviews Online, 27(6), 27-3552-27-3552. https://doi.org/10.5860/choice.27-3552

Han, X., Han, X., Luo, Q., Jacobs, S., \& Jean-Baptiste, M. (2013). Report of a mental health survey among Chinese international students at Yale University. Journal of American College Health, 61(1), 1-8.

Huan, V. S., Ang, R. P., Chong, W. H., \& Chye, S. (2014). The Impact of Shyness on Problematic Internet Use: The Role of Loneliness. The Journal of Psychology, 148(6),

699-715. https://doi.org/10.1080/00223980.2013.825229

Jun, E., SangminChoi. (2015). Academic stress and Internet addiction from general strain theory framework. Computers in Human Behavior, 49, 282-287. https://doi.org/10.1016/j.chb.2015.03.001

Kardefelt-Winther, D. (2014). Problematizing excessive online gaming and its psychological predictors. Computers in Human Behavior, 31, 118-122. https://doi.org/10.1016/j.chb.2013.10.017

Kleim, B., \& Gonzalo, A., D.Ehlers. (2011). The Depressive Attributions Questionnaire (DAQ): Development of a Short Self-Report Measure of Depressogenic Attributions. Journal of Psychopathology and Behavioral Assessment, 33(3), 375-385. https://doi.org/10.1007/s10862-011-9234-9

LaRose, R., \& Eastin, M. S. (2002). A Social Cognitive Explanation of Internet Uses and Gratifications: Toward a New Theory of Media Attendance. International Communication Association.

Lazarus, S., Richard SFolkman. (1984). Stress, appraisal, and coping. Springer Pub. Co.

Luten, A. G., Ralph, J. A., \& Mineka, S. (1997). Pessimistic attributional style: Is it specific to depression versus anxiety versus negative affect? Behaviour Research and Therapy, 35(8), 703-719. https://doi.org/10.1016/s00057967(97)00027-2

Mesidor, J. K., \& Sly, K. F. (2015). Factors that contribute to the adjustment of international students. Journal of International Students, 6(1), 262-282.

Misra, L. G., RanjitaCastillo. (2004). Academic Stress Among College Students: Comparison of American and International Students. International Journal 
of Stress Management, 11(2), 132-148. https://doi.org/10.1037/10725245.11.2.132

Morahan-Martin, P., JanetSchumacher. (2003). Loneliness and social uses of the Internet. Computers in Human Behavior, 19(6), 659-671. https://doi.org/10.1016/s0747-5632(03)00040-2

Morrison, C. M., \& Gore, H. (2010). The Relationship between Excessive Internet Use and Depression: A Questionnaire-Based Study of 1,319 Young People and Adults. Psychopathology, 43(2), 121-126. https://doi.org/10.1159/000277001

Nanyang Technological University. (2016). NTU Education: Vibrant student life. Singapore: Nanyang Technological University. Retrieved from http://www.ntu.edu.sg

Peplau, L. A., \& Perlman, D. (1979). Blueprint for a social psychological theory of loneliness. In Love and attraction: An interpersonal conference (pp. 101110). Pergamon Press New York.

Rice, K. G., Choi, C. C., Zhang, Y., Morero, Y. I., \& Anderson, D. (2012). SelfCritical Perfectionism, Acculturative Stress, and Depression Among International Students. The Counseling Psychologist, 40(4), 575-600. https://doi.org/10.1177/0011000011427061

Russell, D. W. (1996). UCLA Loneliness Scale (Version 3): Reliability, Validity, and Factor Structure. Journal of Personality Assessment, 66(1), 20-40.

Sa, J., Seo, D.-C., Nelson, T. F., \& Lohrmann, D. K. (2013). Cigarette Smoking Among Korean International College Students in the United States. Journal of American College Health, 61(8), 454-467.

Shaw, M., \& Black, D. W. (2008). Internet addiction. CNS Drugs, 22(5), 353-365.

Smith, R. A., \& Khawaja, N. G. (2011). A review of the acculturation experiences of international students. International Journal of Intercultural Relations, 35(6), 699-713. https://doi.org/10.1016/j.ijintrel.2011.08.004

Stoliker, B. E., \& Lafreniere, K. D. (2015). The influence of perceived stress, loneliness, and learning burnout on university students' educational experience. College Student Journal, 49(1), 146-160.

Teo, T. S., Lim, V. K., \& Lai, R. Y. (1997). Users and uses of the internet: The case of Singapore. International Journal of Information Management, 17(5), 325-336. https://doi.org/10.1016/s0268-4012(97)00014-5

Thurber, C., \& Walton, E. A. (2012). Homesickness and Adjustment in University Students. Journal of American College Health, 60(5), 415-419. https://doi.org/10.1080/07448481.2012.673520

VandeCreek, T. L., LeonJackson. (2000). Innovations in clinical practice. Professional Resource Press.

Wang, L. H., \& Wang, L. (2009). A survey on the generalized problematic internet use in Chinese college students and its relations to stressful life events and coping style. International Journal of Mental Health Addiction, 7(2).

Wei, M., Ku, T.-Y., Russell, D. W., Mallinckrodt, B., \& Liao, K. Y.-H. (2008). Moderating effects of three coping strategies and self-esteem on perceived discrimination and depressive symptoms: A minority stress model for Asian international students. Journal of Counseling Psychology, 55(4), 451-462. https://doi.org/10.1037/a0012511 
Wu, H., Garza, E., \& Guzman, N. (2015). International Student's Challenge and Adjustment to College. Education Research International.

Young, K. S. (1998). Internet Addiction: The Emergence of a New Clinical Disorder. CyberPsychology \& Behavior, 1(3), 237-244. https://doi.org/10.1089/cpb.1998.1.237

Young, K. S., \& Rogers, R. C. (1998). The Relationship Between Depression and Internet Addiction. CyberPsychology \& Behavior, 1(1), 25-28. https://doi.org/10.1089/cpb.1998.1.25

OINDRILA DUTTA, Master of Arts in Counselling and Guidance, is currently a doctoral candidate in the School of Humanities and Social Sciences, Nanyang Technological University. She is at present studying grief and bereavement in parents of terminally ill children as well as strategies for addressing such parents' psycho-social-emotional needs and concerns. Email: oindrila001@e.ntu.edu.sg

STEFANIE Y. L. CHYE, PhD, is an Assistant Professor at the Psychological Studies Academic Group, National Institute of Education, Nanyang Technological University. Her research interests include exploring the role of technology and issues related to cyber-wellness. Email: stefanie.chye@nie.edu.sg 